\title{
Measurement Reliability of Axial Length of the Human Eye by using Partial Coherence Interferometry
}

\author{
Jae-hyung Kim, Tae Hwan Moon, Ju Byung Chae, and Sungmin Hyung* \\ Department of Ophthalmology, Chungbuk National University College of Medicine, Cheongju 361-711, Korea
}

(Received July 1, 2014 : revised August 28, 2014 : accepted September 16, 2014)

\begin{abstract}
To investigate the minimum near-infrared ray intensity required (quantifiable threshold value) for consistent measurements of axial length (AL) using partial coherence interferometry (PCI), we attached two polarizing lenses (PL) to two types of PCI (IOLmaster, ALscan). The near-infrared ray intensity of PCI was modified by rotating the axis of one PL at intervals of 5 degrees. The right eye of each volunteer was measured three times and the $\mathrm{AL}$ and signal-to-noise ratio (SNR) was recorded five times for each measurement. Reduction of light intensity was theoretically estimated using Malus' Law. AL was measured consistently with both IOLmaster and ALscan until they reached 55 degrees (1.33\% of intensity) and 60 degrees $(0.77 \%)$, respectively ( $\mathrm{P}=0.343$, Log-rank test). In contrast, SNR decreased as light intensity decreased. In addition, to analyze media opacities that precluded measurement of AL, we retrospectively reviewed the medical records of patients unmeasurable by PCI (ALscan) from May to November 2013. Thirty-eight of 473 eyes (8.0\%) could not be measured using ALscan due to media opacities, such as severe posterior subcapsular cataract (PSC, 11 eyes), hypermature cataract (9 eyes), and vitreous hemorrhage (18 eyes). The mean grades of vitreous haze and PSC were $7.72 \pm 0.96$ and $4.45 \pm 1.04$, respectively. In conclusion, up to $0.77-1.33 \%$ of near-infrared rays decreased, and AL could be measured consistently.
\end{abstract}

Keywords : Axial length, Malus' law, Media opacity, Partial coherence interferometry, Polarizing lens OCIS codes : (170.1610) Clinical applications; (170.4470) Ophthalmology; (170.4460) Ophthalmic optics and devices

\section{INTRODUCTION}

Accurate measurement of ocular axial length (AL) is the most important step for accurate intraocular lens (IOL) power calculations. [1] Accordingly, partial coherence interferometry (PCI) is commonly used to measure ocular AL. PCI provides more accurate, reproducible, and non-contact measurements of ocular AL compared with conventional contact ultrasound methods. [2] PCI uses a laser diode (LD) in the near-infrared spectrum $(780 \mathrm{~nm})$ with a high resolution $(12 \mu \mathrm{m})$ and precision $(0.3-10 \mu \mathrm{m})$ along the fixation line of an eye.[3, 4] The LD in PCI produces coaxial dual beams and mirrors split them into two equal coaxial beams which reflect the beams into the eye (Fig. 1).[5, 6] The measurement mirror moves with constant speed and superimposes the reflected beams from the retina onto the delayed beams from the cornea. $[5,6]$ Then the interference signal is detected and the displacement of the mirror can be precisely obtained. $[5,6]$ The delay of dual beam components equals the axial length. $[5,6]$

However, $\mathrm{PCI}$ is less useful in measuring $\mathrm{AL}$ in eyes with severe media opacity, especially due to posterior subcapsular cataracts (PSC). It is reported that the failure rate of $\mathrm{AL}$ measurement using PCI was $4.7-36.6 \%$ because of dense nuclear sclerosis, PSC, macular pathology, vitreous opacity, and other similar disorders. [7-10] These failures could arise from using devices from different manufacturers, different software versions, differences in regional or hospital/practice settings, etc. [10]

Related to this, unsuccessful measurements of PCI in eyes with severe media opacities meant that the near-infrared light beam centered at $780 \mathrm{~nm}$ could not penetrate the opacities. Although we commonly knew the severities of opacity using the Lens Opacities Classification System III (LOCS

\footnotetext{
*Corresponding author: smh@chungbuk.ac.kr
} 


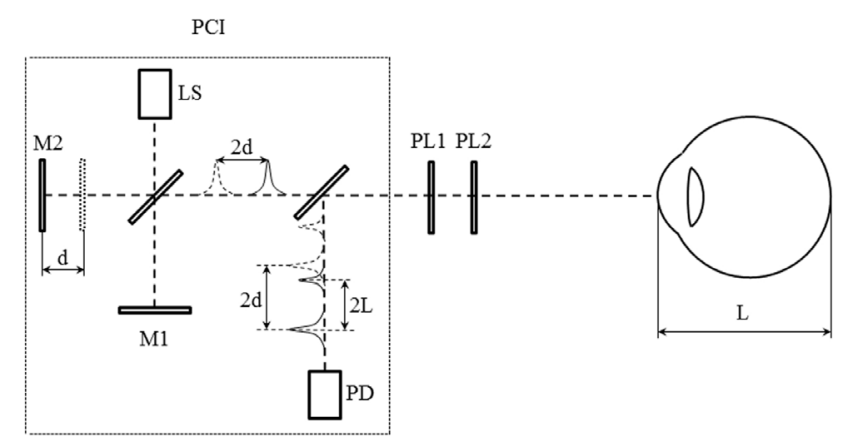

FIG. 1. Schematic diagram of partial coherence interferometry (PCI) and experimental setup using polarizing lens (PL) to attenuate light intensity. Coaxial dual beams produced by PCI are reflected from the eye and superimposed. The interference signal is detected when the delay of dual beam components equals the axial length. In this experiment, two PLs are attached to the measuring part of the PCI. The near-infrared ray intensity of the PCI is modified by rotating the axis of PL2 at intervals of 5 degrees. LS: light source; M1; reference mirror; M2: measurement mirror; PD; photo-detector; L: axial length (Adapted from W. Drexler, O. Findl, R. Menapace, G. Rainer, C. Vass, C. K. Hitzenberger, A.F. Fercher, "Partial Coherence Interferometry: A Novel Approach to Biometry in Cataract Surgery," Am J Ophthalmol. 126, 524-534 (1998))

III) or other grading systems, no studies have assessed the minimum near-infrared ray intensity required for constant PCI measurements of AL. Some studies revealed that increased cataract density could affect the measurements of ocular AL and postoperative refractive outcomes. [11, 12] However, studies were also carried out including eyes that were successfully measured.

We therefore measured the minimum near-infrared ray intensity required for consistent measurements of $\mathrm{AL}$ to quantify a threshold value at which the measurement of $\mathrm{AL}$ became unreliable for PCIs. We also analyzed media opacities in patients with media opacities that precluded measurement using PCI.

\section{METHODS}

\subsection{Measurement of the Minimum Near-infrared Ray Intensity}

Two commercially available types of PCI, IOLmaster 500 (Carl Zeiss Meditec, Dublin, CA) and ALscan (Nidek Technologies, Gamagori, Japan) were used. Two polarizing lenses (PL, Kenko Tokina, Tokyo, Japan) were attached to the measuring part of the PCI. The near-infrared ray intensity of the PCI was modified by rotating the axis of one PL at intervals of 5 degrees. AL and signal-to-noise ratio (SNR) were recorded five times for each measurement and were provided by each PCI. The right eyes of healthy volunteers with no previous ocular disease were measured three times. Reduction of light intensity was theoretically estimated using
Malus' Law. The double path nature of the device which attenuated the light twice was considered to calculate the reduction of light intensity. The minimum near-infrared ray intensity was recorded as the intensity of the near-infrared ray that was calculated from the maximum angle between the two PLs offering constant measurements of ocular AL. The difference of AL according to the attenuation of light intensity was defined as the subtraction of $\mathrm{AL}$ in the uncrossed state from the crossed states. The percentage of SNR was defined as the percentage of the measured SNR in the crossed state over the SNR in the uncrossed state.

\subsection{Analysis of Media Opacities Precluding Successful Measurement using PCI}

We retrospectively reviewed the medical records of patients unmeasurable by ALscan from May to November 2013. The following parameters were analyzed: preoperative visual acuity (logarithm of the minimum angle of resolution, $\log$ MAR); lens opacity graded as LOCS III [13]; and vitreous opacity graded using the vitreous haze scale for photographic grading of vitreous haze in uveitis from 0 (none) to 8 , with approximately $0.3 \log$ step between each step. [14]

\subsection{Statistical Analysis}

Data are presented as means \pm standard deviations, unless otherwise specified in the text. Differences were analyzed with PASW software version 18.0 (SPSS, Chicago, IL). Measurability of PCI was compared using Kaplan-Meier survival curves and the log-rank test. SNR was analyzed using linear regression. The Mann-Whitney $U$ test and the Wilcoxon signed-rank test were used to compare independent and pairs of dependent sample groups, respectively. The Kruskal-Wallis test with modified Bonferroni correction and the chi-squared test were used for more than two dependent sample groups. Two-sided $P$ values $\leq 0.05$ were considered statistically significant.

\section{RESULTS}

\subsection{Measurement of the Minimum Near-infrared Ray Intensity}

Seven right eyes from seven subjects were recruited. The average age was $34.0 \pm 6.2$ years and the average $\mathrm{AL}$ was $25.21 \pm 1.69 \mathrm{~mm}$ with ALscan and $25.20 \pm 1.68 \mathrm{~mm}$ with IOLmaster. AL was measured consistently with both ALscan and IOLmaster, until they reached 60 degrees $(0.77 \%$ of intensity) and 55 degrees ( $1.33 \%$ of intensity), respectively. The differences of AL retained near zero, which meant consistent measurements of AL were done until they reached 0.77 to $1.33 \%$ of near-infrared ray intensity of the PCI (Fig. 2A). There were some missing values due to an inability to measure $\mathrm{AL}$ under $1.33 \%$ of intensity using IOLmaster. However, there were no statistical differences in the measurability between the two types of PCI (Fig. 


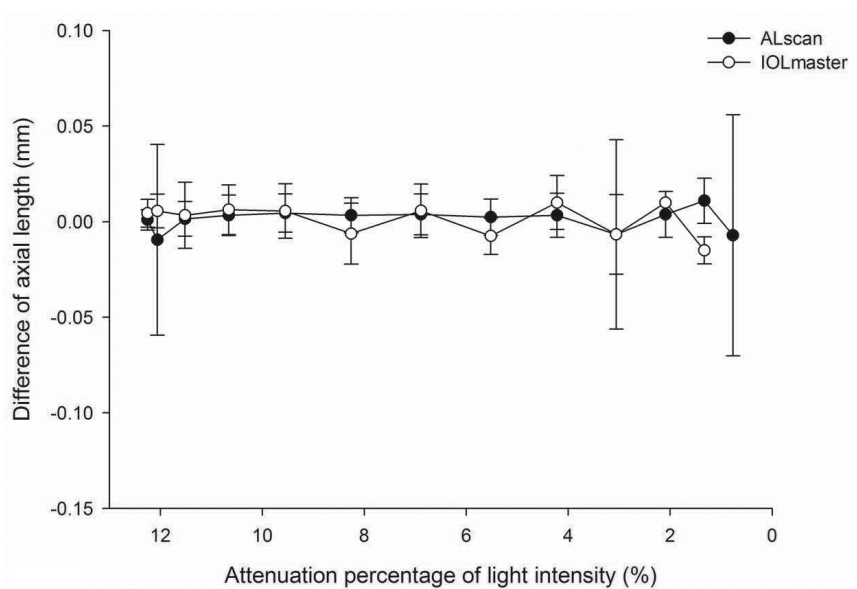

(a)

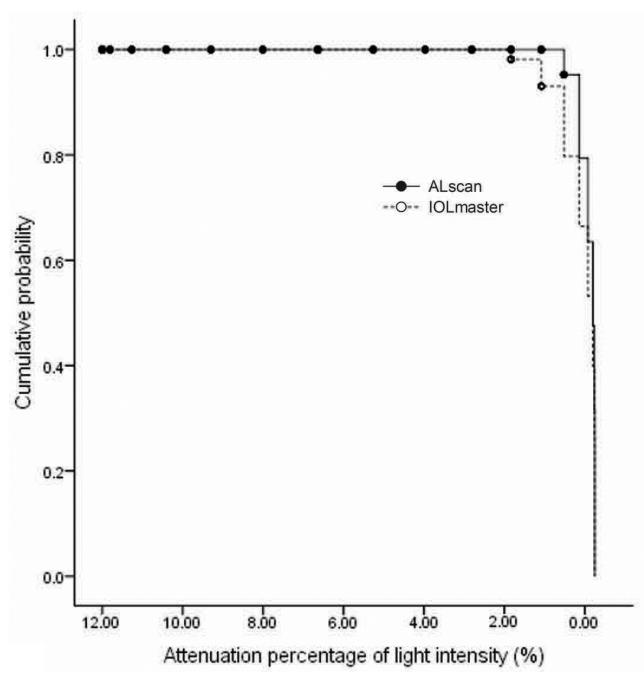

(b)

FIG. 2. Measurability of two partial coherence interferometers (PCIs, ALscan, Nidek Technologies and IOLmaster 500, Carl Zeiss Meditec) according to the attenuation of light intensity. The difference of axial length (AL) was defined as the subtraction of AL in the uncrossed state from that in the crossed states to reduce the light intensity. The differences of AL retained near zero which meant consistent measurements of AL were done until they reached 0.77 to $1.33 \%$ of near-infrared ray intensity of the PCI. There were some missing values using IOLmaster because AL could not be measured under $1.33 \%$ of intensity using IOLmaster. However, there were no statistical differences in the measurability between the two types of PCI (Figure 2B, $P=0.17$, Log-rank test).

TABLE 1. Preoperative data of patients who failed to be measured

\begin{tabular}{c|c|c|c|c}
\hline \hline & $\begin{array}{c}\text { Vitreous Hemorrhage } \\
(\mathrm{n}=18)\end{array}$ & $\begin{array}{c}\text { Dense PSC } \\
(\mathrm{n}=11)\end{array}$ & $\begin{array}{c}\text { Hypermature Cataract } \\
(\mathrm{n}=9)\end{array}$ & $P$ value \\
\hline Mean age (yr) & $60.94 \pm 10.67$ & $67.27 \pm 15.51$ & $61.33 \pm 15.65$ & 0.27 \\
\hline M : F & $11: 7$ & $4: 7$ & $7: 2$ & 0.16 \\
\hline VA (logMAR)* & $2.47 \pm 0.78$ & $1.24 \pm 0.80$ & $2.48 \pm 0.82$ & $<0.01$ \\
\hline Grade of media opacity & $7.72 \pm 0.96$ & $4.45 \pm 1.04$ & White cataract & \\
\hline
\end{tabular}

*: $P$ value $<0.017$, Kruskal-Wallis test with modified Bonferroni correction

VA: visual acuity

PSC: posterior subcapsular opacity

logMAR: logarithm of the minimum angle of resolution

2B, $P=0.17$, Log-rank test). In contrast, SNR decreased as light intensity decreased (Figure 3 ). The results showed a statistically significant correlation between light intensity and SNR in IOLmaster $\left(\mathrm{R}^{2}=0.75, P<0.001\right.$, linear regression) and in ALscan $\left(\mathrm{R}^{2}=0.70, P<0.001\right)$.

\subsection{Analysis of Media Opacities Precluding Successful Measurement using PCI}

Measurement data of 473 eyes in 237 patients using ALscan were reviewed. AL could not be measured in 38 of 473 eyes (failure rate, $8.03 \%$ ). The mean age of the patients was $62.87 \pm 13.36$ years and the average of the best corrected visual acuity (BCVA, logMAR) was $2.12 \pm$ 0.96. Media opacities causing failure of $\mathrm{AL}$ measurements were vitreous hemorrhage (VH, 18 eyes), severe PSC (11 eyes), and hypermature cataract ( 9 eyes). The mean grades of vitreous haze and PSC (LOCS III) were $7.72 \pm 0.96$ and $4.45 \pm 1.04$, respectively. There were no statistical differences in age and gender among the different causes for opacities (Table 1). However, the visual acuity in eyes with dense PSC was better than that in eyes with other opacity causes $(P$ value $<0.017$, Kruskal-Wallis test with modified Bonferroni correction).

\section{DISCUSSION}

In previous studies, the failure rates using PCI and the precludable opacities were reported as $4.7-36.6 \%$. [7-10, 15] However, there were no reports with the objective of comparing the minimum intensity of opacity that could offer successful measurements as a threshold value to measure the $\mathrm{AL}$ accurately. Our results showed that $\mathrm{AL}$ could be measured consistently when up to $0.77-1.33 \%$ of near-infrared 
rays passed through. We used two easily obtainable PLs and Malus' Law, which is one of the well-known principles describing the relationship between polarization and the intensity of the light that passes through an optical material. [16] Because we used inexpensive and easily obtainable materials, the measurability test could be easily reproduced. We used Malus' Law and theoretically assumed the intensity of near-infrared rays that passed through, which might overestimate the minimum intensity of light required. However, it was in good agreement with measured data when the incident light is partially polarized. [17]

Another consideration was the corneal birefringence that might affect the intensity when rotating the axis of the attached PL. [18-20] The cornea is the major birefringent ocular structure, and corneal birefringence varies depending on the location and the orientation. [18] To rule out the effect of orientational variation of corneal birefringence, we also measured $\mathrm{AL}$ while rotating one PL at intervals of 5 degrees. There were no differences in measuring $\mathrm{AL}$ using PCI according to the axis of the PL from 0 to 180 degrees (data not shown). Although conducting measurements three times per axis might mask any possible minimal differences, these differences would be meaningless in the clinical use of PCI.

Successful measurements could be obtained with up to $0.77-1.33 \%$ intensity of the near-infrared rays. Measurements obtained using two different PCIs showed similar results. Figure 2 shows that the differences of AL between the $\mathrm{AL}$ in the uncrossed state and that in the crossed states retained near zero which means consistent measurements of $\mathrm{AL}$ until they reached 0.77 to $1.33 \%$ of near-infrared ray intensity of the PCI. We considered the minimum light intensity with reliable data of $\mathrm{AL}$ as a threshold value of measurement. This is the first report to investigate the measurability of PCI with a concept of measurement threshold value. Clinically, this meant that the patients with failed $\mathrm{AL}$ measurements could have opacities which blocked or scattered more than $99 \%$ of the light at the center of the optical axis of the eye. IOLmaster could not measure under an intensity of $1.33 \%$, which seemed to be higher than those using ALscan $0.77 \%$. However, there were no statistically significant differences between the two PCIs in the measurability using Kaplan-Meier survival curves and in the differences of $\mathrm{AL}$ according to the attenuation of light intensity. The SNRs using both PCIs decreased as light intensity decreased, as expected. The measured range of SNR was different between two PCIs. The different range of SNR would come from the use of different algorithms, devices from different manufacturers, etc. Therefore, we also analyzed the percentage of SNR according to the attenuation of light intensity and they showed significant correlations.

One of the commercially available PCIs, ALscan was recently introduced by Nidek Technologies, and there are no reports of the failure rate using this PCI. Our results show a failure rate of $8.03 \%$. Although patients with vitreous hemorrhage or severe cataract visited our hospital as a

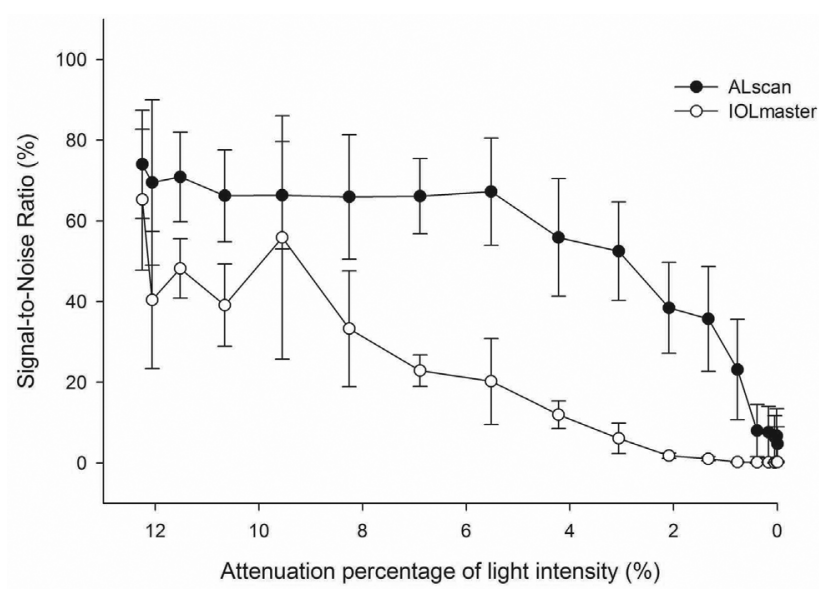

FIG. 3. Changes of the signal-to-noise ratio (SNR) according to the decrease in measured infrared intensity. SNR decreased as light intensity decreased in both PCIs. There was a statistically significant correlation between light intensity and $\mathrm{SNR}$ in IOLmaster ( $\mathrm{R}^{2}=0.75, P<0.001$, linear regression) and in ALscan $\left(\mathrm{R}^{2}=0.70, P<0.001\right)$.

tertiary referral center in our consolidated city-county region, the failure rate was not as high when compared to previous reports using IOLmaster. [7-10] Because of the limitations of retrospective chart review, we could not obtain repeated measurements of $\mathrm{AL}$ using two PCIs, which could have shown whether measurability between two PCIs might differ or not,in clinical aspects. However, we inferred similar results between the two PCIs from our objective measurements of minimum intensity that offered consistent $\mathrm{AL}$.

\section{CONCLUSION}

We revealed the minimum intensity of infrared ray (0.77-1.33\%) for consistent measurement of AL using PCI. This could be considered as a quantifiable threshold of $\mathrm{AL}$ measurement. This was the first report to investigate the quantifiable measurability of PCI with a concept of measurement threshold. As a clinical aspect, this study showed that the patients with failed AL measurements could have opacities which blocked or scattered more than $99 \%$ of the light at the center of the optical axis of the eye. We also reported the failure rate of a recently introduced PCI. Though there were differences between visible and infrared light, we expect that our results will provide some insight into investigating the visual impacts of opacities in an eye according to their density and location.

\section{ACKNOWLEDGMENT}

This work was supported by the research grant of Chungbuk National University in 2013. 


\section{REFERENCES}

1. T. Olsen, "Sources of error in intraocular lens power calculation," J. Cataract Refract Surg. 18, 125-9 (1992).

2. O. Findl, W. Drexler, R. Menapace, B. Kiss, C. K. Hitzenberger, and A. F. Fercher, "Optical biometry in cataract surgery," Dev. Ophthalmol. 34, 131-40 (2002).

3. O. Findl, W. Drexler, R. Menapace, C. K. Hitzenberger, and A. F. Fercher, "High precision biometry of pseudophakic eyes using partial coherence interferometry," J. Cataract Refract Surg. 24, 1087-93 (1998).

4. A. R. Wissa, S. S. Wahba, and M. M. Roshdy, "Agreement and relationship between ultrasonic and partial coherence interferometry measurements of axial length and anterior chamber depth," Clin Ophthalmol. 6, 193-8 (2012).

5. W. Drexler, O. Findl, R. Menapace, G. Rainer, C. Vass, C. K. Hitzenberger, and A. F. Fercher, "Partial coherence interferometry: a novel approach to biometry in cataract surgery," Am. J. Ophthalmol. 126, 524-34 (1998).

6. J. Santodomingo-Rubido, E. A. Mallen, B. Gilmartin, and J. S. Wolffsohn, "A new non-contact optical device for ocular biometry,” Br. J. Ophthalmol. 86, 458-62 (2002).

7. Y. T. Hsieh and I. J. Wang, "Intraocular lens power measured by partial coherence interferometry," Optom. Vis. Sci. 89, 1697-701 (2012).

8. Y. A. Chen, N. Hirnschall, and O. Findl, "Evaluation of 2 new optical biometry devices and comparison with the current gold standard biometer," J. Cataract Refract Surg. 37, 513-7 (2011).

9. P. J. Buckhurst, J. S. Wolffsohn, S. Shah, S. A. Naroo, L. N. Davies, and E. J. Berrow, "A new optical low coherence reflectometry device for ocular biometry in cataract patients," Br. J. Ophthalmol. 93, 949-53 (2009).

10. N. Hirnschall, S. Murphy, D. Pimenides, V. Maurino, and O. Findl, "Assessment of a new averaging algorithm to increase the sensitivity of axial eye length measurement with optical biometry in eyes with dense cataract," J. Cataract Refract Surg. 37, 45-9 (2010).
11. T. Ueda, H. Ikeda, T. Ota, T. Matsuura, and Y. Hara, "Relationship between postoperative refractive outcomes and cataract density: Multiple regression analysis," J. Cataract Refract Surg. 36, 806-9 (2010).

12. T. Ueda, F. Taketani, T. Ota, and Y. Hara, "Impact of nuclear cataract density on postoperative refractive outcome: IOL master versus ultrasound," Ophthalmologica 221, 384-7 (2007).

13. L. T. Chylack, Jr., J. K. Wolfe, D. M. Singer, M. C. Leske, M. A. Bullimore, I. L. Bailey, J. Friend, D. McCarthy, and $\mathrm{S}$. Y. $\mathrm{Wu}$, "The lens opacities classification system III. The longitudinal study of cataract study group," Arch Ophthalmologica 111, 831-6 (1993).

14. J. L. Davis, B. Madow, J. Cornett, R. Stratton, D. Hess, V. Porciatti, and W. J. Feuer, "Scale for photographic grading of vitreous haze in uveitis," Am. J. Ophthalmol. 150, 637-641 e1 (2010).

15. T. S. Dietlein, G. Roessler, C. Luke, S. Dinslage, S. Roters, P. C. Jacobi, P. Walter, and G. K. Krieglstein, "Signal quality of biometry in silicone oil-filled eyes using partial coherence laser interferometry," J. Cataract Refract Surg. 31, 1006-10 (2005).

16. W. Li, X. Lu, and Y. Lin, "Novel absolute displacement sensor with wide range based on malus law," Sensors (Basel) 9, 10411-22 (2009).

17. I. Damian, "Malus' law for a real polarizer," arXiv preprint physics/0604073 (2006).

18. K. Irsch and A. A. Shah, "Birefringence of the central cornea in children assessed with scanning laser polarimetry," J. Biomed Opt. 17, 086001 (2012).

19. R. N. Weinreb, C. Bowd, D. S. Greenfield, and L. M. Zangwill, "Measurement of the magnitude and axis of corneal polarization with scanning laser polarimetry," Arch Ophthalmol. 120, 901-6 (2002).

20. R. W. Knighton and X. R. Huang, "Linear birefringence of the central human cornea," Invest Ophthalmol. Vis Sci. 43, 82-6 (2002). 